\title{
Long-term prophylactic intravenous antibiotics after Kasai portoenterostomy for biliary atresia do not reduce the risks of post-operative cholangitis, a retrospective study
}

\author{
Xi-Si Guan^, Qiu-Ming He^, Wei Zhong^, Jia-Kang Yu^, Zhe Wang^ \\ Department of Neonatal Surgery, Guangzhou Women and Children's Medical Center, Guangzhou, China \\ Contributions: (I) Conception and design: XS Guan, Z Wang; (II) Administrative support: W Zhong, JK Yu; (III) Provision of study materials or \\ patients: XS Guan; (IV) Collection and assembly of data: Z Wang; (V) Data analysis and interpretation: Z Wang, QM He; (VI) Manuscript writing: \\ All authors; (VII) Final approval of manuscript: All authors. \\ Correspondence to: Zhe Wang, MD. Guangzhou Women and Children's Medical Center, 9 Jinsui Road, Guangzhou 510623, China. \\ Email: wonderzhe@hotmail.com.
}

Background: Postoperative cholangitis (PC) is the most common and serious complication of biliary atresia (BA) patients post-Kasai portoenterostomy (KPE). The duration of prophylactic intravenous antibiotics (IVA) after KPE varies with no clear consensus. We conducted a retrospective cohort study to explore the effects of IVA duration on preventing post-operative cholangitis and analyze the risk factors for cholangitis and short-term prognosis.

Methods: All patients diagnosed with BA and received KPE in Guangzhou Women and Children's Hospital in 2018, were included in this study. The patients received prophylactic IVA after KPE. Firstly, the patients were divided into two groups based on the presence or absence of PC (PC and NPC group). The correlation between PC and the IVA duration was analyzed, followed by a comparison of short-term prognosis, outcome, and other risk factors between the groups. Next, the patients were divided based on the median IVA duration of 11 days (long IVA and short IVA group), followed by a comparison of the incidence of PC, short-term prognosis, outcome, and other risk factors between the two groups.

Results: Totally 89 patients were included in this study. Amount them, eleven patients who were lost during follow-up, were excluded from the study. The prophylactic IVA duration of the PC $(n=52)$ and NPC $(n=25)$ groups was $12.6 \pm 8.5$ and $13.0 \pm 4.5$ days, respectively $(\mathrm{P}=0.79)$. Further, the jaundice clearance rate of the two groups was similar (PC: $31 / 52$, NPC: $13 / 25, \mathrm{P}=0.53$ ). There was no difference in the incidence and frequency of cholangitis between the short $(n=42)$ and long $(n=35)$ IVA groups $(27 / 42,25 / 35, P=0.51)$, and the duration of IVA had no effect on jaundice clearance $(24 / 42,20 / 35, \mathrm{P}=1.00)$. The short IVA group had a significantly shorter hospital stay than the long IVA group $\left(16.2 \pm 5.1,25.3 \pm 8.3, \mathrm{P}=8.95 \times 10^{-8}\right)$. Patients undergoing KPE at an older age were at a higher risk of cholangitis (NPC: $60.6 \pm 19.7$, PC: $72.3 \pm 17.8, \mathrm{P}=0.01$ ).

Conclusions: A long duration of IVA after KPE for BA may not be necessary. Early diagnosed patients had timely surgery had a lower incidence of PC. Our findings may help in promoting the scientific use of antibiotics and reducing the LHS.

Keywords: Postoperative cholangitis (PC); intravenous antibiotics (IVA); biliary atresia (BA); Kasai portoenterostomy (KPE); length of hospital stay (LHS)

Submitted Jun 17, 2021. Accepted for publication Aug 17, 2021.

doi: 10.21037/tp-21-309

View this article at: https://dx.doi.org/10.21037/tp-21-309

\footnotetext{
^ ORCID: Xi-Si Guan, 0000-0001-7014-2416; Zhe Wang, 0000-0001-9116-9722; Qiu-Ming He, 0000-0002-0365-1648; Wei Zhong, 0000-00028163-3269; Jia-Kang Yu, 0000-0002-7634-3275.
} 


\section{Introduction}

Biliary atresia (BA) is the most common cause of obstructive jaundice in infants. It manifests as progressive obstruction of both the intrahepatic and extrahepatic bile ducts, leading to cholestasis and hepatic fibrosis. The incidence ranges from 1:5,000 to 1:19,000 of live births (1). Currently, Kasai portoenterostomy (KPE) is the preferred treatment for BA. However, there have been cases where even after $\mathrm{KPE}$, patients still developed progressive liver fibrosis and eventually required liver transplantation. If untreated, progressive liver cirrhosis can lead to death by the age of two years.

Postoperative cholangitis (PC) is the most common and serious complication of KPE, occurring in $40 \%$ to $93 \%$ of patients' post-surgery (2). Although the mechanism of PC has not been fully established, limited evidence indicates that PC is mainly caused by poor bile drainage, ascending bacterial infection, intestinal microecological changes, and certain other factors. The occurrence and frequency of PC are closely related to the prognosis of BA. Several studies have shown that the earlier cholangitis occurs after KPE, the worse the prognosis is (3). Furthermore, the occurrence of PC is related to the native liver survival rate (NLSR) (4).

Pediatric surgeons have been taking active measures to prevent $\mathrm{PC}$ after $\mathrm{KPE}$, including long bile limb reservation $(10 \mathrm{~cm} / \mathrm{kg})$ and anti-reflux valve construction (5). The prophylactic use of intravenous antibiotics (IVA) after surgery is a common practice; however, there is no consensus on which antibiotic is the most appropriate or the duration it should be withheld. Most medical centers prefer a broad-spectrum antibiotic cover of gram-negative bacilli and enterococci (6). The 2018 Chinese Medical Association (CMA) BA treatment consensus suggest using IVA for at least two weeks after KPE; however, there is currently no high-level evidence to support this protocol $(7,8)$. The CMA consensus about IVA preventing $\mathrm{PC}$ is based on a prospective non-randomized clinical controlled trial published in 2003 by Meyers et al. (9). In this trail, the authors used IVA for 8-12 weeks to prevent infection risks caused by the steroids, however, they did not compare the incidence of PC between groups in their trail and made no comments on the effect of long term IVA. Internationally, some centers in the United States withhold IVA until the patient begins feeding (usually 48 hours after the surgery) and then subsequently convert IVA to long-term oral antibiotics (10). On the other hand, Meyers et al. suggested 8-12 weeks' IVA after KPE would improve bile flow (9).
In European centers, IVA is used during the perioperative period (11). However, in some Japanese centers, postoperative IVA continues for a relatively long time, up to a few weeks or more (12).

There is limited information on the relationship between the duration of postoperative IVA and the incidence of PC after KPE in BA patients. Long-term prophylactic postoperative IVA not only prolongs the hospital stay, but also increases the risk of antibiotic resistance, Is it the longer, the better? We conducted a retrospective cohort study to explore the effects of IVA duration on preventing post-operative cholangitis after KPE and analyze the risk factors for cholangitis and short-term prognosis. We present the following article in accordance with the STROBE reporting checklist (available at https://dx.doi. org/10.21037/tp-21-309).

\section{Methods}

\section{Patients}

This is a retrospective cohort study. All patients with BA treated by KPE surgery at the Guangzhou Women and Children's Medical Center (GWCMC) between January 1st, 2018, and December 31st, 2018, were included. The patients included were followed up for one year or until the endpoint (liver transplantation or death), patients failed to follow the protocol or lost during follow-up were excluded. All the operations were performed by the same medical team in the same manner: the triangular cord was dissected to the liver capsule behind the portal vein at both corners, a $10 \mathrm{~cm} / \mathrm{kg}$ bile limb was constructed with a $5-\mathrm{cm}$ antireflux valve which was created by sewing the input and bile limb together at the mesentery and contralateral mesentery margins with 5-0 non-absorbable polypropylene sutures. Patients were followed in clinic and by phone call. During the first 6 months, patients visit the BA clinic once a month, after the first 6 months, they were connected by third party social media software privately or by phone call and visit clinic every 6 months. Written informed consent for each patient was signed by at least one parent, and the study was approved by the Ethics Committee of Guangzhou Women and Children's Medical Center (ID of the approval: [2020]01701). The study conformed to the provisions of the Declaration of Helsinki (as revised in 2013). GWCMC is the South China National Medical Center, shares the second largest BA population in China mainland. Leading professors in GWCMC participates in the preparation of 
CMA BA expert consensus.

\section{Diagnosis of PC}

PC was diagnosed if the clinical manifestations met both of the following criteria: (I) a complete blood count suggesting bacterial infection (high C-reactive protein levels, and/ or an increased count or percentage of neutrophils) or the presence of fever (body temperature higher than $37.5^{\circ} \mathrm{C}$ ) that could not be explained by other reasons; (II) a light stool color and/or abrupt recurrence or increase in clinical jaundice with increased direct bilirubin. The occurrence of PC, frequency of PC, and time interval (days) from KPE to the first onset of $\mathrm{PC}$ was recorded during the follow-up period.

\section{Prophylactic use of IVA}

Postoperative IVA administration was withheld between 3 and 48 days, depending on the clinical course and attending surgeon. The same protocol was implemented for all patients. One therapeutic dose of third-generation cephalosporin (cefoperazone-sulbactam, CPZ-S) was administered intravenously to the patients $30 \mathrm{~min}$ before KPE. If surgery extended beyond 3 hours, the patient would receive an additional dose of CPZ-S. After surgery, $\mathrm{CPZ}-\mathrm{S}$ was combined with the intravenous administration of metronidazole, which lasted for a certain number of days. After withdrawal of IVA, oral antibiotics that consisted of cefaclor alternating with sulfamethoxazole once a week were administered to the patients. Oral antibiotics were continued for six months post-KPE.

\section{Patient groups and statistical analyses}

Firstly, the patients were divided into two groups based on PC occurrence: a cholangitis group (PC group) and a noncholangitis group (NPC group). The mean duration of prophylactic IVA in the groups was compared. Further, the patients were regrouped based on the median IVA duration of 11 days (long IVA group, >11 days; short IVA group, $<11$ days). The incidence of PC, frequency of PC, and interval from KPE to the first onset of PC were compared for these two groups. Other factors such as age at KPE, length of hospital stay (LHS), jaundice clearance rate (JCR), mortality, liver transplantation rate (LTR), and native liver survival rate (NLSR) were also analyzed. Jaundice clearance was defined by total bilirubin (TBiL) $\leq 25 \mu \mathrm{mol} / \mathrm{L}$.
Persistent JC was defined by TBiL remaining $\leq 25 \mu \mathrm{mol} / \mathrm{L}$ at the end of the follow-up.

Data are presented as the mean \pm standard error or percentage. Categorical data were analyzed by the chisquare test or Fisher exact test, and continuous data were analyzed by unpaired $t$-tests or nonparametric tests (the Mann-Whitney test). All analyses were performed with GraphPad Prism version 6.00 for Windows (GraphPad Software, San Diego, California, USA). Data signifies significance with $\mathrm{P}<0.05$.

\section{Results}

\section{Outcomes}

A total of 89 patients diagnosed with BA underwent KPE during the study period. Of these patients, 77 cases were followed up for one year or until the endpoint (liver transplantation or death), while 11 cases were lost, and these latter cases were excluded from the study. The mean age on admission and at KPE was $61.0 \pm 20.0$ and $68.9 \pm 18.3$ days, respectively. The IVA duration after KPE ranged from 3 to 49 days (13.4 46.6 days). The mean LHS was $21.2 \pm 9.9$ days. During the one-year follow-up, PC occurred in $68 \%$ of the patients (52/77). The average interval between the KPE and onset of the first PC was $65.9 \pm 44.7$ days, ranging from 7 to 187 days, with a median of 60 days. The mean frequency of PC was $3.0 \pm 2.3$ episodes, ranging from 1 to 10 episodes, with a median of 2.5 episodes. The JCR at the end of three months was $57 \%$ (44/77), and the persistent JCR was $48 \%$ (37/77). The TBiL of these patients was normal at the end of the follow-up period. By the end of the follow-up, a total of 19 patients had received liver transplantation (LTR: $24.7 \%$ ), and the average age at liver transplantation was $8.1 \pm 3.2$ months. Two patients died during the follow-up. The overall 1-year NLSR was $72.7 \%$.

\section{Risk factors for $P C$}

The age of the patient on admission and at KPE, LHS, IVA duration, short-term outcomes, and long-term outcomes were compared between the PC group $(\mathrm{n}=52)$ and NPC group $(\mathrm{n}=25)$ (Table 1). The mean duration of prophylactic IVA was $12.6 \pm 8.5$ and $13.0 \pm 4.5$ days for the PC and NPC groups, respectively, and this difference was not statistically significant $(\mathrm{P}=0.79)$. However, patients in the NPC group were significantly younger than those in the $\mathrm{PC}$ group both on admission $(54.2 \pm 20.3$ vs. $64.2 \pm 20.2, \mathrm{P}=0.05)$ and at $\mathrm{KPE}$ 
Table 1 Comparisons between the NPC and PC groups

\begin{tabular}{lccc}
\hline Clinical features & $\mathrm{NPC}(\mathrm{n}=25)$ & $\mathrm{PC}(\mathrm{n}=52)$ & $\mathrm{P}$ \\
\hline Age on admission (days) & $54.2 \pm 20.3$ & $64.2 \pm 20.2$ & 0.05 \\
Age at Kasai (days) & $60.6 \pm 19.7$ & $72.3 \pm 17.8$ & 0.01 \\
LHS (days) & $18.0 \pm 6.7$ & $21.4 \pm 8.5$ & 0.08 \\
IVA duration (days) & $12.6 \pm 8.5$ & $13.0 \pm 4.5$ & 0.79 \\
JC & 13 & 31 & 0.53 \\
Persistent JC & 12 & 25 & 0.99 \\
LTx & 6 & 13 & 0.20 \\
Death & 1 & 1 & 0.89 \\
NLSR & $72.0 \%$ & $73.1 \%$ & 0.92
\end{tabular}

Italic $P$ values indicate statistically significant. IVA, intravenous antibiotics; LHS, length of hospital stay; PC, postoperative cholangitis; NPC, No postoperative cholangitis; JC, jaundice clearance; LTx, liver transplantation; NLSR, native liver survival rate.

$(60.6 \pm 19.7$ vs. $72.3 \pm 17.8, \mathrm{P}=0.01)$. The LHS, short-term outcomes, and long-term outcomes showed no differences between the two groups.

\section{Effect of the duration of prophylactic IVA}

Patients were divided into short IVA $(\mathrm{n}=42)$ and long IVA $(n=35)$ groups based on the median duration of 11 days of IVA (Table 2). There were no statistical differences in the incidence of PC (27/42 vs. 25/35, $\mathrm{P}=0.51), \mathrm{KPE}$ to first PC interval (57.6 \pm 39.2 vs. $75.4 \pm 49.2, \mathrm{P}=0.16$ ), or the frequency of $\mathrm{PC}(3.0 \pm 2.5$ vs. $2.9 \pm 2.1, \mathrm{P}=0.86)$ between the two groups. Age on admission, age at KPE, and the outcomes also showed no differences. The LHS was significantly shorter in the short IVA group than in the long IVA group $(16.2 \pm 5.1$ vs. $\left.25.3 \pm 8.3, \mathrm{P}=9.0 \times 10^{-8}\right)$. Then the patients were divided in short $(n=51)$ or long IVA group $(n=26)$ based on the 14 days of IVA according to the CMA BA consensus (Table 3). Same as above, there were no statistical differences in the incidence of PC (32/51 vs. 20/26, $\mathrm{P}=0.2)$, KPE to first PC interval $(60.1 \pm 44.4$ vs. $75.8 \pm 44.4, \mathrm{P}=0.23)$ or the frequency of $\mathrm{PC}(3.2 \pm 2.4$ vs. $2.7 \pm 2.1, \mathrm{P}=0.42)$ between the two groups. Age on admission, age at KPE, and the outcomes also showed no differences.
Table 2 Comparisons between the short and long IVA groups (divided based on the median duration of 11 days of IVA)

\begin{tabular}{lccc}
\hline Clinical features & $\begin{array}{c}\text { Short IVA } \\
(\leq 11 \mathrm{~d})(\mathrm{n}=42)\end{array}$ & $\begin{array}{c}\text { Long IVA } \\
(>11 \mathrm{~d})(\mathrm{n}=35)\end{array}$ & $\mathrm{P}$ \\
\hline Age on admission (days) & $61.4 \pm 20.7$ & $60.4 \pm 20.9$ & 0.83 \\
Age at Kasai (days) & $67.7 \pm 20.2$ & $69.4 \pm 17.9$ & 0.70 \\
LHS (days) & $16.2 \pm 5.1$ & $25.3 \pm 8.3$ & $9.0 \times 10^{-8}$ \\
Occurrence of PC & 27 & 25 & 0.51 \\
KPE to 1st PC (days) & $57.6 \pm 39.2$ & $75.4 \pm 49.2$ & 0.16 \\
Frequency of PC & $3.0 \pm 2.5$ & $2.9 \pm 2.1$ & 0.86 \\
JC & 24 & 20 & 1.00 \\
Persistent JC & 21 & 16 & 0.71 \\
LTx & 12 & 7 & 0.38 \\
Death & 1 & 1 & 0.89 \\
NLSR & $69.0 \%$ & $77.1 \%$ & 0.43 \\
\hline
\end{tabular}

Italic $\mathrm{P}$ value indicates statistically significant. IVA, intravenous antibiotics; LHS, length of hospital stay; PC, postoperative cholangitis; JC, jaundice clearance; LTX, liver transplantation; NLSR, native liver survival rate.

Table 3 Comparisons between the short and long IVA groups (divided based on the 14 days IVA according to the CMA BA consensus)

\begin{tabular}{lccc}
\hline Clinical features & $\begin{array}{c}\text { Short IVA } \\
(<14 \mathrm{~d})(\mathrm{n}=42)\end{array}$ & $\begin{array}{c}\text { Long IVA } \\
(\geq 14 \mathrm{~d})(\mathrm{n}=26)\end{array}$ & $\mathrm{P}$ \\
\hline Age on admission (days) & $59.7 \pm 21.6$ & $63.3 \pm 18.8$ & 0.47 \\
Age at Kasai (days) & $66.6 \pm 20.0$ & $72.2 \pm 17.1$ & 0.22 \\
LHS (days) & $17.2 \pm 6.5$ & $26.4 \pm 7.6$ & $5.2 \times 10^{-7}$ \\
Occurrence of PC & 32 & 20 & 0.2 \\
KPE to 1st PC (days) & $60.1 \pm 44.4$ & $75.8 \pm 44.4$ & 0.23 \\
Frequency of PC & $3.2 \pm 2.4$ & $2.7 \pm 2.1$ & 0.42 \\
JC & 27 & 17 & 0.18 \\
Persistent JC & 24 & 13 & 0.81 \\
LTx & 16 & 3 & 0.06 \\
Death & 1 & 1 & 0.62 \\
NLSR & $67.0 \%$ & $84.6 \%$ & 0.09 \\
\hline
\end{tabular}

Italic $\mathrm{P}$ value indicates statistically significant. IVA, intravenous antibiotics; LHS, length of hospital stay; PC, postoperative cholangitis; JC, jaundice clearance; LTX, liver transplantation; NLSR, native liver survival rate. 


\section{Discussion}

$\mathrm{PC}$ is the most common complication after KPE in patients with BA; it can alter the short-term and long-term outcomes. Many studies have examined the risk factors of cholangitis development in patients with KPE. Ernest van Heurn et al. suggested that cholangitis may be related to bile outflow, where the risk of early cholangitis was significantly higher in patients with partial postoperative bile flow compared to those with good or poor postoperative bile flow (2). Li et al. found incidences of cholangitis were closely related to the preoperative nutritional status of patients. Patients with malnutrition were more susceptible to the development of early cholangitis, and the episodes of cholangitis were more frequent (13). Various preventive measures have been taken against the occurrence of PC; one such common practice is the prophylactic use of IVA after KPE surgery. However, there is no consensus on either the most favorable antibiotic or the duration of its administration. The related protocols vary dramatically in different centers $(9,11,12)$.

In this retrospective study, short-term postoperative prophylactic IVA was not a risk factor for PC occurrence. The IVA duration in the PC group was not significantly different compared to that of the NPC group. Liu et al. compared 119 PC patients and 61 NPC patients in their study; they also found that IVA duration was not significantly different between PC vs. NPC groups, early $v s$. late PC groups, or single vs. recurrent PC groups (14). In the present study, when the patients were regrouped into long-term and short-term IVA groups based on the 11-day median cut-off, the PC incidence was similar in both groups. This finding also indicates that a short IVA duration is not related to an increase in PC occurrence. The 2018 Tokyo Guidelines on antimicrobial therapy for acute cholangitis and cholecystitis suggest that antibiotics should not be used for more than 4-7 days even after severe bile tract infection. This suggestion is based on the evidence that prolonged use of antibiotics does not decrease the risk of recurrent infection (15). Furthermore, prolonged antibiotic administration will alter the intestinal flora composition, creating a potential risk of bile infection. As a result of these findings, the appropriateness of long-term prophylactic IVA after KPE should be reconsidered.

According to the results of our study, the incidence of cholangitis is related to patient age at the time of surgery; the older the patient at the time of KPE, the higher the incidence of cholangitis. Many studies have reported that the patient's age at the time of surgery is a significant factor influencing the surgical outcome (16). Kasai et al. have reported a better surgical outcome in infants younger than 60 days of age (17). Data from the Japanese Biliary Atresia Registry show an inverse correlation between the age at portoenterostomy and JCR (18). Limited studies have demonstrated the relationship between age at surgery and cholangitis. Liu et al. determined that older patients undergoing surgery had a higher risk of recurrent cholangitis. In their study, a larger body mass before KPE, which might also indicate an older patient, served as a risk factor for cholangitis (14). Usually, an older patient could be expected to have a greater degree of liver fibrosis, which could lead to incomplete bile drainage, thus increasing the risk of PC. However, the underlying mechanism of this aspect needs further targeted research.

The prognostic indicators in the short- and longterm IVA groups are similar in our study. This finding suggests that short-term administration of IVA may not only be effective but also have no adverse effect on patient outcomes. Additionally, the LHS was significantly shorter in the short-term IVA group than in the long-term group. Therefore, a short-term IVA protocol may be a more cost-effective and efficient strategy in the postoperative management of BA and more beneficial for patients and their families.

The present study had several limitations. First, the sample size of this study is relatively small, so negative results on IVA-PC relationship may be due to the lack of statistical power. Second, it was a relatively short-term follow-up for patients with BA. Therefore, we needed further follow-up for our surviving patients to valid to long-term result. Third, the study was conducted in a single center, the conditional outcome might not be generalizable to the majority of patients with BA in whole country. Future research should consider utilizing data from multiple centers.

\section{Conclusions}

This study showed no difference in the incidence, frequency, and time of onset of cholangitis between the short and long IVA groups. Also, the duration of IVA did not affect jaundice clearance and NLSR. Furthermore, older patients had a higher risk of cholangitis. Based on these findings, it is recommended that the duration of IVA and shortening of the LHS should be attempted. The risk of PC may be reduced through early diagnosis and timely KPE. 


\section{Acknowledgments}

Funding: This work was supported by the Guangzhou Health Science and Technology project, General guidance project 20201A011033.

\section{Footnote}

Reporting Checklist: The authors have completed the STROBE reporting checklist. Available at https://dx.doi. org/10.21037/tp-21-309

Data Sharing Statement: Available at https://dx.doi. org/10.21037/tp-21-309

Conflicts of Interest: All authors have completed the ICMJE uniform disclosure form (available at https://dx.doi. org/10.21037/tp-21-309). The authors have no conflicts of interest to declare.

Ethical Statement: The authors are accountable for all aspects of the work in ensuring that questions related to the accuracy or integrity of any part of the work are appropriately investigated and resolved. Written informed consent for each patient was signed by at least one parent, and the study was approved by the Ethics Committee of Guangzhou Women and Children's Medical Center (ID of the approval: [2020]01701). The study conformed to the provisions of the Declaration of Helsinki (as revised in 2013).

Open Access Statement: This is an Open Access article distributed in accordance with the Creative Commons Attribution-NonCommercial-NoDerivs 4.0 International License (CC BY-NC-ND 4.0), which permits the noncommercial replication and distribution of the article with the strict proviso that no changes or edits are made and the original work is properly cited (including links to both the formal publication through the relevant DOI and the license). See: https://creativecommons.org/licenses/by-nc-nd/4.0/.

\section{References}

1. Hartley JL, Davenport M, Kelly DA. Biliary atresia. Lancet 2009;374:1704-13.

2. Ernest van Heurn LW, Saing H, Tam PK. Cholangitis after hepatic portoenterostomy for biliary atresia: a multivariate analysis of risk factors. J Pediatr 2003;142:566-71.
3. Koga H, Wada M, Nakamura H, et al. Factors influencing jaundice-free survival with the native liver in postportoenterostomy biliary atresia patients: results from a single institution. J Pediatr Surg 2013;48:2368-72.

4. Qiao G, Li L, Cheng W, et al. Conditional probability of survival in patients with biliary atresia after Kasai portoenterostomy: a Chinese population-based study. J Pediatr Surg 2015;50:1310-5.

5. Nio M, Wada M, Sasaki H, et al. Technical standardization of Kasai portoenterostomy for biliary atresia. J Pediatr Surg 2016;51:2105-8.

6. Baumann U, Ure B. Biliary atresia. Clin Res Hepatol Gastroenterol 2012;36:257-9.

7. Neonatal Surgery Group PHSG, Pediatric Surgery Branch, Chinese Medical Association. Diagnosis and treatment of biliary atresia in mainland China (expert consensus). Chinese Journal of Pediatric Surgery 2013;34:700-5.

8. Pediatric Organ Transplantation Group and Hepatobiliary Surgery Group PSB. Chinese Medical Association Guidelines for diagnosing \& treating biliary atresia (2018 Edition). Journal of Clinical Hepatology 2019;35:2435-40.

9. Meyers RL, Book LS, O'Gorman MA, et al. High-dose steroids, ursodeoxycholic acid, and chronic intravenous antibiotics improve bile flow after Kasai procedure in infants with biliary atresia. J Pediatr Surg 2003;38:406-11.

10. Cowles RA. The Jaundiced Infant: Biliary Atresia. In: Pediatric Surgery (Seventh Edition). Volume 2, 7 edn. Edited by Coran AG. United States of America: Mosby, 2012:1327.

11. Kelly DA, Davenport M. Current management of biliary atresia. Arch Dis Child 2007;92:1132-5.

12. Nio M, Ohi R. Biliary atresia. Semin Pediatr Surg 2000;9:177-86.

13. Li D, Chen X, Fu K, et al. Preoperative nutritional status and its impact on cholangitis after Kasai portoenterostomy in biliary atresia patients. Pediatr Surg Int 2017;33:901-6.

14. Liu J, Dong R, Chen G, et al. Risk factors and prognostic effects of cholangitis after Kasai procedure in biliary atresia patients: A retrospective clinical study. J Pediatr Surg 2019;54:2559-64.

15. Gomi H, Solomkin JS, Schlossberg D, et al. Tokyo Guidelines 2018: antimicrobial therapy for acute cholangitis and cholecystitis. J Hepatobiliary Pancreat Sci 2018;25:3-16.

16. Nio M, Wada M, Sasaki H, et al. Effects of age at Kasai portoenterostomy on the surgical outcome: a review of the literature. Surg Today 2015;45:813-8. 
17. Kasai M, Watanabe I, Ohi R. Follow-up studies of long term survivors after hepatic portoenterostomy for "noncorrectible" biliary atresia. J Pediatr Surg 1975;10:173-82.

Cite this article as: Guan XS, He QM, Zhong W, Yu JK, Wang Z. Long-term prophylactic intravenous antibiotics after Kasai portoenterostomy for biliary atresia do not reduce the risks of post-operative cholangitis, a retrospective study. Transl Pediatr 2021;10(8):2076-2082. doi: 10.21037/tp-21-309
18. Nio M. Japanese Biliary Atresia Registry. Pediatr Surg Int 2017;33:1319-25.

(English Language Editor: D. Fitzgerald) 